\title{
REVUE
}

\section{EMPLOI DU CAOUTCHOUC DANS L'INDUSTRIE LAITIËRE}

\author{
par \\ G. GÉNIN \\ Ingénieur E. P. C.
}

Les champs d'application du caoutchouc se sont considérablement développés au cours de ces dernières années et en particulier l'industrie des produits alimentaires fait un appel de plus en plus important à cette matière première. Les services d'hygiène ont même été dans l'obligation d'édicter, dans certains cas, des règlements concernant la composition du caoutchouc destiné à être placé au contact des produits alimentaires, afin d'éviter que la présence des constituants normaux des mélanges du caoutchouc ne puisse altérer la pureté de ces produits.

On sait, en effet, que le caoutchouc manufacturé n'est pas constitué de gomme pure, mais qu'il contient différentes substances, tels que soufre, matières colorantes, oxyde de zinc, accélérateurs de vulcanisation, ete., dont les caractères chimiques sont parfois tels qu'ils pourraient rendre dangereuse la consommation des produits alimentaires placés à leur contact.

Voici par exemple quelques extraits des législations étrangères concernant ce point particulier. Au Danemark et en Allemagne, la législation applicable au caoutchoue destiné à être au contact des produits alimentaires remonte à quelques années déjà. Dans les autres pays, les règlements sont plus récents et par exemple, dans presque tous les pays, le plomb doit être absolument exclu des mélanges de caoutchouc employés par les industries alimentaires. Le zinc est interdit dans les pays suivants : Danemark, Suède et Suisse. L'antimoine en Autriche, en Belgique, au Canada et en Allemagne. En fin, l'arsenic est naturellement absolument interdit, mais les fabricants de caoutchouc sont parfaitement documentés sur ce point et n'ont jamais cherché à introduire des composés arsénieux dans le caoutchoue manufacturé, utilisé par les industries alimentaires.

L'industrie laitière utilise le caoutchouc sous deux formes principales, soit sous la forme de latex, e'est-à-dire du lait qui s'écoule des arbres à caoutchouc, lorsque ceux-ci sont incisés, soit sous la forme de caoutchouc souple et vulcanisé.

EMPLOIS DU LATEX DANS L'INDUSTRIE LAITIÈRE

C'est surtout aux Etats-Unis que le latex est utilisé comme produit de scellement des boîtes de conserves. Le but du latex est de 
constituer une pellicule plastique que l'on introduit au eours de la fabrication des boittes de conserve, en particulier de lait en poudre ou de lait condensé, entre la partie latérale de la boîte d'une part et le fond ou le couvercle d'autre part, afin de compenser les petites irrégularités du fer-blane. Théoriquement, il serait impossible sans soudure d'obtenir un joint parfait, si la présence de cette pellieule de caoutchouc n'était prévue.

Pendant très longtemps, on a utilisé dans ce but des solutions de caoutchouc dans le benzène, mais l'emploi de ces solutions présentait des dangers d'incendie et on ne pouvait manipuler facilement les solutions renfermant plus de $8 \%$ de eaoutchouc, de sorte que la pellicule de caoutchouc ainsi interposée était trop mince. C'est en 1902 que l'on voit pour la première fois décrit l'emploi de ces joints de caoutchouc. Le premier brevet cependant n'a été accordé qu'en 1914 à Milne (Brevet anglais 24.680). Immédiatement après la guerre, la firme américaine Dewey and Almy Chemical Company entreprit ses recherches sur l'emploi du latex, et obtint des résultats tels que le latex a rapidement supplanté les solutions de caoutehouc dans le benzène. Parmi les avantages qui résultent de l'emploi du jatex, il faut citer la suppression des dangers d'incendie, la non toxicité du latex, la possibilité d'obtenir une pellicule de caoutchoue plus épaisse et plus résistante, et enfin la possibilité d'appliquer les produits de scellement au latex sur des tôles de fer blanc vernies. Les progrès dans cette direction ont été tels qu'on estime qu'en 1930, il a été fabriqué suivant ce procédé aux Etats-Unis plus de 4.500.000.000 de boîtes. Ce n'est toutefois qu'après de nombreuses recherches sur le choix de la concentration du latex et de sa vitesse d'évaporation que l'on est parvenu à un résultat aussi satisfaisant. Il faut en effet que les propriétés des produits de scellement à base de latex soient rigoureusement contrôlées, quand on songe qu'il existe aux Etats-Unis des machines fabriquant environ 400 boites à la minute. Cette application du latex a été protégée dans les brevets anglais 196.881 de $1924,302.587$ de 1928 , et 306.040 de 1929 .

Indiquons également qu'on a breveté aux Etats-Unis (Brevet. américain 1.664.635 de 1928) l'emploi du latex comme produit de scellement pour le collage des disques en carton qui bouchent les bouteilles de lait.

\section{EMPLOI DU CAOUTCHOUC SOUPLE}

Le caoutchoue souple est un des matériaux les plus importants entrant dans la constitution des machines à traire. Il sert à revêtir le godet trayeur et à constituer la gaine élastique de ce godet et est employé pour la confection des tuyaux flexibles qui relient les godets trayeurs au réservoir à lait. Le caoutchouc employé pour constituer 
la gaine élastique doit être suffisamment mou et élastique pour permettre la fixation des godets sur les trayons de différentes dimensions sans blesser ou incommoder l'animal. La surface intérieure doit être parfaitement unie et non striée, car les stries permettraient l'introduction de bulles d'air qui entraîneraient un sûrissement rapide du lait.

Il n'a pas été imposé de spécifications concernant la qualité du caoutchoue utilisé pour la fabrication de ces gaines élastiques. On admet toutefois que la teneur en caoutchouc doit varier de 70 à $90 \%$. Ces gaines sont formées soit par boudinage et vuleanisation sur des mandrins en aluminium, soit par moulage (India Rubber $J$., t. XLVIII, p. 1003, 1914 et t. LXXXII, p. 563, 1931). La dureté varie de 35 à $45^{\circ}$ au duromètre Shore.

Ces objets doivent également présenter une résistance aussi élevée que possible aux matières grasses du lait; or on exige d'autre part une grande plasticité du caoutchouc, et ce sont là deux conditions opposées ; aussi les difficultés dans la fabrication de ces objets en caoutchouc sont-elle très grandes. Les études entreprises dans de nombreux pays ont permis de les surmonter en partie.

L'absorption de la matière grasse du lait par le caoutchouc rend très importante la question du nettoyage et de la stérilisation des machines à traire. Si après chaque opération, les appareils sont rincés à l'eau ou avec une solution de savon, et si les parties en caoutchouc sont ensuite plongées dans l'eau bouillante pendant un temps suffisant pour qu'il y ait stérilisation, le caoutchoue est transformé au bout de 2 semaines en une masse gonflée, collante, qui possède une odeur désagréable et qui peut renfermer jusqu'à $20 \%$ de matière grasse extraite du lait. Un autre procédé de nettoyage quisemble préférable, consiste à démonter complètement l'appareil, afin de séparer les parties en caoutchouc qui sont immergées pendant toute une nuit dans une solution de soude caustique à $15 \%$, puis trempées dans l'eau bouillante pendant une heure. Ce procédé forme des savons par réaction de l'alcali sur la matière grasse et il y a en même temps stérilisation des objets.

Le Ministère de l'Agriculture des Etats-Unis s'est occupé officiellement de cette question du nettoyage et de la stérilisation des machines à traire (Farmers' Bull., mars 1923, p. 1315). D'après ce Bulletin, si, après chaque traite, on place les parties en caoutchouc dans de l'eau portée à une température comprișe entre 70 et $75^{\circ}$, et si on laisse ensuite la température s'abaisser lentement, la durée moyenne des gaines élastiques des godets trayeurs est de 3 semaines, celle des cuves servant au transport du lait d'environ 24 semaines, et celle des cuves reliant l'appareil à la machine à vide d'environ 31 semaines. Pratiquement, voici comment il faut opérer : après 
chaque traite, les machines sont rincées à l'eau froide ou à l'eau légèrement tiède. Les gaines élastiques et les tubes sont alors rincés avec de l'eau, contenant en solution les produits de lavage employés normalement pour stériliser les appareils de la laiterie. Après rinçage dans l'eau froide, on plonge les objets en eaoutchoue dans l'eau à $75^{\circ}$. Toutefois, on peut porter la durée des gaines de 3 à 23 semaines, en effectuant la stérilisation comme suit. Après un séjour des parties en caoutchouc de 20 à 24 minutes dans l'eau chaude, on les immerge dans une solution diluée de chlore ou on les place dans un réfrigérateur ou encore on les met soigneusement à l'abri des mouches et autres insectes dans un local très propre. La solution de chlore est préparée en dissolvant 1 gramme de solution standard dans environ 13 litres d'eau ; la solution standard est elle-même préparée en dissolvant 370 grammes de chlorure de chaux dans 4-5 litres d'eau froide.

Parfitt (Ind. Agr, Expt. Stat. Rept. p. 32, 1930) a élevé quelques objections à l'emploi du chlore comme désinfectant, En particulier, lorsqu'on utilise des eaux dures pour la préparation de ces solutions de chlore, il se forme un dépôt sableux granulaire qu'il est très difficile d'éliminer des parties en caoutchoue de la machine. On peut corriger cet inconvénient en ajoutant au chlorure de chaux une certaine quantité de phosphate trisodique. De même, le chlore entraînerait un craquellement du caoutchouc. D'après cet auteur, une solution a $0,3 \%$ de soude caustique serait aussi efficace que les solutions de chlore.

L'emploi đu caoutchouc dans la fabrication des tétines peut être considéré comme une application de ce produit dans l'industrie laitière. Les seuls points qu'il importe de signaler dans cette application sont ceux qui sont relatifs au choix des matériaux employés dans la fabrication de ces tétines. Lorsqu'on ne disposait que du caoutchoue para pour la fabrication des objets en eaoutchouc, il était courant d'ajouter à ce produit des factices blanes ou bruns, et comme colorants, on employait du sulfure mercurique. La vuleanisation des tétines se faisait à froid par immersion dans une solution de chlorure de soufre dans le sulfure de carbone, soit à chaud dans les vapeurs de chlorure de soufre. Le traitement final consistait en un lavage dans une solution chaude de carbonate de soude, dans un rinçage à l'eau froide, puis dans un traitement superficiel au moyen de glycérine (Gummi Ztg., 1912, t. XXVII, p. 207 ; India Rubber $J$., 1912, t. XLIV, p. 1298). Nous rappellerons qu'en France, il a été prohibé, vers 1912-1913, la vente des tétines qui ne seraient pas préparées en partant de caoutchoue pur vulcanisé à chaud. Dans certains pays étrangers et en particulier en Allemagne, on a protesté contre cette législation, et deux auteurs Frank et 
MarCKWALD (Gummi Ztg., 1913, t. XXVII, p. 1428) ont assuré que toutes les tétines fabriquées industriellement eontenaient des succédanés, que la vuleanisation au chlorure de soufre en solution donnait un produit nullement dangereux, et que les solvants étaient complètement chassés de la tétine avant la fin de la fabrication.

On a également étudié la question de savoir si le sulfure d'antimoine utilisé comme colorant pouvait être la cause d'un danger par suite de son extraction du caoutchouc. Esch a montré (Bull. Sciences Pharmacol., 1922, t. XXIX, p. 121 ; Gummi Ztg., 1922, t. XXXVI, p. 1298) que s'il est possible d'extraire de petites quantités d'antimoine par traitement des tétines avec une solution chaude et concentrée d'alcali, ces conditions ne correspondent nullement aux conditions d'emploi des tétines et la salive et le lait n'ont aucune activité comparable à celle des solutions caustiques. Cet auteur a indiqué qu'en Allemagne encore les tétines sont pigmentées avec du sulfure mercurique, que dans certains pays elles sont traitées avant leur vente par une solution alcaline afin de dissoudre l'excès de soufre libre, puis vernies et que dans ces conditions, on a exagéré les risques d'empoisonnement par l'antımoine.

Ces quelques exemples montrent l'importance des applications du caoutchoue dans l'industrie laitière et les problèmes intéressants posés par l'emploi de ce produit au contact des substances grasses qui risquent d'en diminuer considérablement les qualités.

\section{BIBLIOGRAPHIE ANALYTIQUE}

\section{LES LIVRES}

Leclainche (E.), Membre de l'Institut. - Histoire de la Mlédecine vétérinaire. Edité par l'Office du Livre, Toulouse, rue Alsace-Lorraine, 42, 1936. Un vol. grand in- $8^{\circ}$ de 812 pages. Prix : 80 francs.

Dans le magnifique et important ouvrage dont il vient de doter la littérature vétérinaire, le Professeur-Leclarnche, membre de l'Académie des Sciences, directeur honoraire du Ministère de l'Agrieulture, directeur de l'Office international des Epizooties, retrace, en une forme pleine d'attrait et de vie, l'histoire de la médecine des animaux depuis ses très lointaines origines jusqu'à nos jours.

Ce livre, d'un puissant intérêt, où l'auteur, savant de haute culture et de forte érudition, affirme des qualités éminentes d'historien et de littérateur, que pourraient lui envier bien des contemporains versés dans les sciences historiques et dans l'art d'écrire, est divisé en deux parties d'inégale àmpleur. La première, la plus courte, s'étend jusqu'à l'époque, 1763, où ont été créés et organisés l'enseignement et les Ecoles vétérinaires et englobe trois des grandes périodes de l'histoire universelle : l'Antiquité, le Moyen-âge et là Renaissance. 